\title{
Lopinavir/ritonavir in the treatment of HIV-I infection: a review
}

\author{
Ashish Chandwani' \\ Jonathan Shuter ${ }^{2}$ \\ 'Division of Infectious Diseases, \\ Montefiore Medical Center \\ and the Albert Einstein College \\ of Medicine, Bronx, NY, USA; ${ }^{2}$ AIDS \\ Center and Division of Infectious \\ Diseases, Montefiore Medical Center \\ and the Albert Einstein College \\ of Medicine, Bronx, NY, USA
}

\begin{abstract}
Lopinavir/ritonavir is the first and only coformulated HIV-1 protease inhibitor (PI). Large clinical trials have demonstrated lopinavir/ritonavir's clinical efficacy in both antiretroviral-naïve and -experienced patients. The immunologic and virologic benefits of treatment with this agent have been proven in HIV-infected adults, adolescents, and children. Smaller studies support the use of lopinavir/ritonavir monotherapy as a therapeutic option in certain patients. The drug is characterized by a high genetic barrier to resistance, and appears to be more forgiving of non-adherence than earlier, unboosted PIs. The most frequent side effects observed are diarrhea, nausea, and vomiting. These gastrointestinal adverse effects are generally mild to moderate. Metabolic derangements, including hyperlipidemia and glucose intolerance, have also been observed in lopinavir/ritonavir recipients. As the menu of available antiretroviral agents continues to expand, lopinavir/ritonavir remains a proven and effective drug for the treatment of HIV infection.
\end{abstract}

Keywords: lopinavir/ritonavir, protease inhibitor, HIV, antiretroviral, Kaletra ${ }^{\circledR}$

Highly active antiretroviral therapy (HAART) has revolutionized the management of HIV-1 infection. Antiretroviral therapy has improved steadily in terms of efficacy, tolerability, and dosing convenience since the advent of HAART in 1995. Lopinavir/ ritonavir (Kaletra ${ }^{\circledR}$ ) was the sixth drug in the HIV-1 protease inhibitor (PI) class to be approved by the US Food and Drug Administration (FDA). Approved in September 2000 in the US (April 2001 in Europe) for the treatment of HIV infection in adults and children older than 6 months of age, it was the first, and only, PI to be coformulated. It has been widely and effectively used in both antiretroviral naïve and experienced HIV-infected patients around the world. The current tablet formulation of lopinavir/ ritonavir was approved by the FDA in October 2005, and the capsule formulation was phased out in the US 6 months later in favor of the new tablet. This article will review the pharmacologic and clinical aspects of lopinavir/ritonavir in the management of HIV infection.

\section{Historical aspects}

The availability of PIs changed the landscape of HIV therapy. Treatment with these agents produced virologic and immunologic improvements not previously seen with the nucleoside reverse transcriptase inhibitors (NRTIs) that were the standard of care (Sham et al 1998). They offered, for the first time, the possibility of long-term survival to persons living with HIV/AIDS (PLWHAs) (Deeks et al 1997; Sham et al 1998; Rana and Dudley 1999). However, all of the early PIs had limited bioavailability, high protein binding, large pill burdens, and short half-lives producing low trough levels (ie, nadir drug levels at the end of a dosing interval) and requiring frequent dosing. They were also associated with significant side effects that compromised adherence, 
and thus promoted the development of drug resistance (Deeks et al 1997; Sham et al 1998). Virologic failure was extremely common in those who did not maintain very high levels of adherence (Paterson et al 2000), and the outlook for patients who failed their first PI-based regimen was bleak (Gulick et al 2000; Hammer et al 2003).

In 1997, Abbott Laboratories unveiled its new protease inhibitor, ABT-378, later to be named lopinavir, at the 4th Conference on Retroviruses and Opportunistic Infections. It was developed in an attempt to retain activity despite mutations in the HIV-1 protease gene. Lopinavir was found to be 3 to 4 times more active against HIV than ritonavir. Administered alone, lopinavir exhibits poor bioavailability. However, its blood levels are dramatically increased by low doses of ritonavir, a potent inhibitor of cytochrome P450 3A4. Coformulated lopinavir/ritonavir was the first combination pill to contain a drug (lopinavir) not available separately. Lopinavir/ritonavir $\left(\right.$ Kaletra $\left.^{\circledR}\right)$ received accelerated FDA approval for the treatment of HIV infection in combination with other agents for both antiretroviral naïve and experienced patients. The approval was based upon analyses of plasma HIV RNA and CD4+ lymphocyte count outcomes in a controlled study of treatment of HIV infection with lopinavir/ritonavir and in smaller uncontrolled doseranging studies of lopinavir/ritonavir of 72 weeks duration (FDA/Center for Drug Evaluation and Research). Lopinavir was not the first PI to take advantage of ritonavir boosting, but the improved efficacy of lopinavir/ritonavir over prior agents (Walmsley et al 2002) ushered in an era in which ritonavir boosting of PI therapy became the norm in HIV management. The original formulation of lopinavir/ritonavir $\left(\right.$ Kaletra $\left.^{\circledR}\right)$ was a soft-gelatin capsule containing $133.3 \mathrm{mg}$ of lopinavir and $33.3 \mathrm{mg}$ of ritonavir, and it required storage in a refrigerator. The recommended adult dosage of the original formulation was 3 capsules taken twice daily (or 6 tablets once daily in antiretroviral-naïve patients). A new tablet formulation of lopinavir/ritonavir which used proprietary melt-extrusion technology (Meltrex ${ }^{\mathrm{TM}}$ ) was approved by the FDA in 2005. The new formulation consisted of a stable, solid dispersion of the drugs within a tablet. The new Kaletra ${ }^{\circledR}$ tablets each contain $200 \mathrm{mg}$ lopinavir and $50 \mathrm{mg}$ ritonavir. This formulation provides a lower pill burden, similar bioavailability, decreased effect of food on absorption of the drug, and an elimination of the refrigeration requirement (Klein et al 2007). In April 2005, the FDA issued an additional approval for once-daily lopinavir/ritonavir in adult patients with no prior antiretroviral treatment. An oral solution of lopinavir/ ritonavir containing $80 \mathrm{mg}$ lopinavir and $20 \mathrm{mg}$ ritonavir per $\mathrm{ml}$ is available and must be taken with food. Since 2007 a lower-dose tablet formulation of lopinavir/ritonavir (100 mg/25 mg) has been available in the US (available in the EU since early 2008) for the pediatric population.

The introduction of lopinavir/ritonavir to the developing world has been accompanied by some controversy. Initiatives begun in recent years to make HAART available in resource poor areas have placed pressure on drug manufacturers to supply their medications at reduced cost. Current World Health Organization (WHO) guidelines recommend lopinavir/ritonavir, boosted fosamprenavir, or boosted atazanavir as the anchor drugs in second line regimens (World Health Organization 2006). WHO notes lopinavir/ritonavir's co-formulation and heat stability as advantages over the

\section{Kaletra (ABT -378/r) co - formulation}
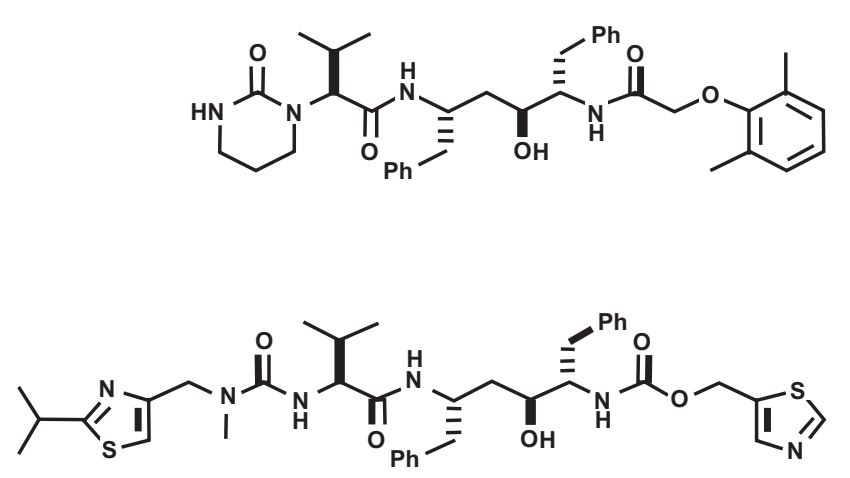

\author{
ABT -378 \\ Active antiviral component \\ $400 \mathrm{mg}$
}

Figure I Molecular structures of lopinavir (ABT-378) and ritonavir (RTV). Courtesy of Abbott Laboratories, with permission. 
other PIs. In the developing world, lopinavir/ritonavir tablets are distributed under the brand name, Aluvia, a formulation that is pharmacologically identical to but visually distinct from Kaletra ${ }^{\circledR}$. Abbott Laboratories has been the target of criticism for not distributing the drug widely enough and for not making it affordable enough (Third World Network 2006). As of April 2007, Abbott has offered lopinavir/ritonavir through its preferential pricing program to all of Africa and some additional "least developed" nations at a price of US\$500 annually per patient, and to 45 "lower income" and "lower middle income" nations at a price of US\$1000 annually per patient. The annual price is considerably higher than that of other anchor drugs, especially nevirapine, but is discounted more than $90 \%$ from the cost in the developed world. To date, the efforts of generic drug manufacturers to develop an equivalent product for a lower price have been unsuccessful.

\section{Pharmacology Pharmacokinetics}

The standard adult dose of lopinavir/ritonavir is $400 \mathrm{mg} / 100$ mg twice daily. The mean peak plasma concentration $\left(\mathrm{C}_{\max }\right)$ at this dose is $9.8 \pm 3.7 \mu \mathrm{g} / \mathrm{mL}$, occurring approximately 4 hours after administration of a dose. A once-daily dosing regimen of $800 \mathrm{mg} / 200 \mathrm{mg}$ may be used in therapy-naïve patients. This regimen is not recommended for therapy experienced patients. The tablets should be swallowed whole, and should not be chewed, crushed, or broken. Food intake does not affect absorption of the tablet formulation. However, the liquid formulation should be taken with food to improve absorption. The absolute bioavailability of lopinavir co-formulated with ritonavir in humans has not been established. At steady state, lopinavir is approximately 98\%-99\% bound to plasma proteins. The mean trough concentration is $5.2 \mu \mathrm{g} / \mathrm{mL}$ and the mean elimination halflife of lopinavir/ritonavir ranges from 2 to 3 hours after a single dose and from 4 to 6 hours after multiple-dose administration. Lopinavir/ritonavir accumulates intracellularly and the intracellular/plasma concentration ratio is 1.18 (Crommentuyn et al 2004). Lopinavir is lipid-soluble and penetrates the cerebrospinal fluid (CSF). It produces significant reductions in the CSF viral load (Yilmaz et al 2004; Letendre et al 2007). Lopinavir undergoes rapid first-pass metabolism in the liver by CYP3A4 and CYP3A5. Ritonavir inhibits the CYP3A4 isoenzyme in the human liver microsomes and results in increased concentrations of lopinavir when the two drugs are coadministered (Kumar et al 1999). Lopinavir/ritonavir is primarily eliminated by the fecal route with urinary excretion accounting for $<2 \%$ of the eliminated drug (Kumar et al 2004).

\section{Mechanism of action}

HIV-1 protease is an aspartic protease that cleaves both structural and functional proteins from precursor viral polypeptide strands and fulfills an essential role in the viral lifecycle. Inhibition of the protease produces immature, non-infectious virions. Lopinavir is a potent inhibitor of HIV-1 protease. The lopinavir/ritonavir coformulation produces its antiviral effect by inhibiting the formation of infectious virions, thus preventing subsequent waves of cellular infection (Flexner 1998).

\section{Use in pregnancy}

Lopinavir/ritonavir is classified as category $\mathrm{C}$ (ie, no adequate and well-controlled studies in pregnant women - should be used only if the potential benefit justifies the potential risk to the fetus) for use in pregnant women. There are no controlled studies evaluating the use of lopinavir/ritonavir in pregnant women with HIV infection. No treatment-related malformations were observed when lopinavir in combination with ritonavir was administered to pregnant rats or rabbits. Developmental anomalies in embryos and fetuses were observed when rats were treated with supratherapeutic doses of lopinavir/ritonavir (Cunha et al 2007). It should be used during pregnancy only when the care provider and patient conclude that the benefits of the drug exceed the potential risks to the fetus. The CDC recommends that HIV-infected mothers should not breastfeed their infants to avoid risking postnatal exposure to HIV. It is not known whether lopinavir is secreted in human milk. In the US, where HIV-infected women are strongly discouraged from breastfeeding, this is of little relevance. In resource poor areas of the world, where breastfeeding is still the norm, the potential implications of neonatal exposure to the drug need to be considered.

\section{Pediatric use}

The safety, efficacy, and pharmacokinetic profiles of lopinavir/ritonavir in pediatric patients below the age of 6 months have not been established. Once-daily dosing of lopinavir/ritonavir has, likewise, not been evaluated in pediatric patients. The recommended dosage in children is $100 \mathrm{mg} / 25 \mathrm{mg}$ to $400 \mathrm{mg} / 100 \mathrm{mg}$ twice daily based upon the body surface area or weight. Before prescribing lopinavir/ritonavir 100/25 mg tablets, children should be assessed for the ability to swallow intact tablets (Abbott Laboratories). 


\section{Use in the setting of hepatic and/or renal insufficiency}

Lopinavir is primarily metabolized by the liver, and drug concentrations may increase in hepatic impairment. Plasma protein binding is also decreased in hepatic impairment. Lopinavir/ritonavir should be used with caution in patients with significant hepatic impairment. Patients with underlying hepatitis B or C or marked elevations in transaminase levels prior to treatment may be at increased risk for developing or worsening of transaminase elevations or hepatic decompensation with use of lopinavir/ritonavir. Hepatitis $\mathrm{C}$ coinfection and baseline elevations of transaminases are associated with an increased risk of severe liver events (Gonzalez-Requena et al 2004; Palacios et al 2006). Lopinavir/ritonavir has very limited excretion through the kidneys, and renal insufficiency has little impact on the pharmacokinetics of the drug (FDA/Center for Drug Evaluation and Research).

\section{Use in the setting of other comorbidities}

New onset diabetes mellitus, exacerbation of pre-existing diabetes mellitus, and hyperglycemia have been reported during post-marketing surveillance in HIV-1 infected patients receiving lopinavir/ritonavir therapy, but a causal relationship has not been established (Abbott Laboratories). It may be prudent to exercise caution in the use of this drug in those with diabetes mellitus or glucose intolerance. Similarly, hyperlipidemia is a relatively common consequence of lopinavir/ritonavir therapy, and this should be considered when therapeutic choices are made for patients with pre-existing lipid disorders.

\section{Clinical trials}

Lopinavir/ritonavir has been extensively studied for use in the management of HIV-infection in both treatment-naïve and treatment-experienced patients. According to the guidelines for the treatment of HIV infection by the US Department of Health and Human Services and British HIV Association, lopinavir/ritonavir is one of the preferred PIs for use in the first-line treatment regimens of HIV-infected adults and adolescents (Panel on clinical practices for treatment of HIV infection; Gazzard B on behalf of BHIVA Writing Committee). It is also a standard of care option in the International AIDS Society-USA Panel recommendations (Hammer et al 2006).

\section{Treatment-naïve patients}

Abbott 720 was a randomized blinded, multi-center trial evaluating treatment with lopinavir/ritonavir at 3 dose levels (200/100 mg twice daily, 400/100 mg twice daily, and 400/200 mg twice daily) together with stavudine (40 mg bid) and lamivudine (150 mg bid). All patients were switched to lopinavir/ritonavir $400 / 100 \mathrm{mg}$ twice daily between weeks 48 and 72. Through 360 weeks of treatment, the proportion of patients with HIV-1 RNA $<400$ copies $/ \mathrm{mL}$ and $<50$ copies $/ \mathrm{mL}$ were $61 \%$ and $59 \%$ respectively $(\mathrm{n}=100)$. Among patients completing 360 weeks of treatment with CD4+ cell count measurements $(n=60)$, the mean (median) increase in CD4+ lymphocyte count was 501 (457) cells $/ \mathrm{mm}^{3}$ above baseline. Thirty-nine patients (39\%) discontinued the study, including 13 (13\%) discontinuations due to adverse reactions and 1 (1\%) death (Murphy et al 2001, 2008). This study established the antiviral efficacy of lopinavir/ritonavir, and the extremely long follow-up provided evidence for the sustained effect of this agent over time.

In a randomized, double-blind, multicenter trial $(n=653)$ comparing lopinavir/ritonavir (400/100 mg twice daily) plus stavudine and lamivudine versus nelfinavir (750 mg 3 times daily) plus stavudine and lamivudine, a significantly larger proportion of patients treated with lopinavir/ritonavir than with nelfinavir achieved the endpoints of $<400$ copies $/ \mathrm{mL}$ of HIV RNA ( $75 \%$ vs $63 \%, \mathrm{p}<0.001)$ and $<50$ copies $/ \mathrm{mL}$ $(67 \%$ vs $52 \%, p<0.001)$ (Walmsley et al 2002). None of the patients treated with lopinavir/ritonavir in this study developed HIV protease mutations, in contrast to $33 \%$ of those treated with nelfinavir $(p<0.001)$. Similar mean increases from baseline CD4+ lymphocyte counts to week 48 were observed in the lopinavir/ritonavir group and the nelfinavir group (207 and 195 cells $/ \mathrm{mm}^{3}$, respectively). This study demonstrated the superiority of lopinavir/ritonavir over unboosted nelfinavir, which was the previous standard of care.

An open-label, multicenter trial compared the efficacy of lopinavir/ritonavir $(800 / 200 \mathrm{mg})$ once daily plus tenofovir DF and emtricitabine versus lopinavir/ritonavir 400/100 mg twice daily plus tenofovir DF and emtricitabine in 190 antiretroviral treatment naïve patients (Murphy et al 2001) $71 \%$ of the patients treated with once daily lopinavir/ ritonavir achieved and maintained virologic suppression (VL $<50$ copies $/ \mathrm{mL}$ ) compared with $65 \%$ of the patients who were treated with a regimen of twice-daily lopinavir/ ritonavir. This study proved that once-daily dosing of lopinavir/ritonavir is therapeutically equivalent to twice-daily dosing in antiretroviral naïve subjects.

KLEAN ( $\mathrm{n}=878$ ) was an open-label, randomized, noninferiority study that compared fosamprenavir-ritonavir with lopinavir-ritonavir, each in combination with a fixed dose of 
abacavir-lamivudine, for 48 weeks (Eron et al 2006). The antiviral and immunological responses in the fosamprenavir/ ritonavir group were similar to those in the lopinavir/ritonavir group with a similar tolerability and adverse effect profile.

The GEMINI study evaluated the efficacy and tolerability of saquinavir/ritonavir versus lopinavir/ritonavir each in combination with emtricitabine/tenofivir as initial therapy in antiretroviral naïve patients over 48 weeks (Walmsley et al 2007). It was a prospective multicenter open labeled trial with 337 patients in 5 countries. At 48 weeks, patients treated with boosted saquinavir showed a similar virologic response when compared to the patients treated with lopinavir/ritonavir with $64.7 \%$ and $63.5 \%$ of patients treated with saquinavir/ritonavir and lopinavir/ritonavir, respectively, achieving VL $<50$ copies/mL. The CD4+ lymphocyte count response in the two groups was also comparable with a median increase from baseline of 178 for the patients treated with saquinavir/ritonavir and 204 for patients treated with lopinavir/ritonavir. The degree of lipid perturbation was higher in the lopinavir/ritonavir group than in the boosted saquinavir group.

The CASTLE trial compared boosted atazanavir and lopinavir/ritonavir in 883 antiretroviral naïve patients. The effect on viral suppression (HIV RNA $<50$ ) was similar at 48 weeks ( $78 \%$ vs $76 \%$ respectively). The mean CD4+ lymphocyte count increases from baseline were 203 cells/ $\mathrm{mm}^{3}$ in atazanavir/ritonavir-treated patients and 219 cells/ $\mathrm{mm}^{3}$ in patients who were treated with lopinavir/ritonavir. However, the patients treated with ritonavir boosted atazanavir demonstrated a lower incidence of gastrointestinal adverse events and hyperlipidemia (Molina et al 2008).

The ARTEMIS study was a randomized trial comparing once daily darunavir/ritonavir to once or twice daily lopinavir/ritonavir in 687 antiretroviral-naïve subjects. At 48 weeks, time-to-loss-of-virologic response analysis determined that $84 \%$ patients receiving darunavir and $78 \%$ patients receiving lopinavir achieved HIV viral RNA $<50$ copies/mL thus proving the non-inferiority of darunavir/ritonavir. Lopinavir/ritonavir was found to have a less favorable safety profile with a greater number of gastrointestinal-related adverse effects (Ortiz et al 2008).

A recent study completed by the AIDS Clinical Trials Group (ACTG), Study A5142, compared regimens of 2 nucleoside/tide reverse transcriptase inhibitors (NRTIs) plus lopinavir ritonavir versus two NRTIs plus efavirenz, versus an NRTI-sparing regimen of efavirenz and lopinavir/ ritonavir in 757 antiretroviral-naïve subjects (Riddler et al 2008). The authors found that, over 96 weeks of follow-up, virologic failure was significantly less frequent in the two NRTI-efavirenz arm than in the two NRTI-lopinavir/ritonavir arm. Increases of CD4+ lymphocyte counts from baseline were significantly higher in the two NRTI-lopinavir/ritonavir arm, and incidence of new drug resistance mutations was lower in the two NRTI-lopinavir/ritonavir arm. The NRTIsparing combination of efavirez and lopinavir/ritonavir produced rates of viral suppression similar to those of two NRTIs-efavirenz, but was associated with increased toxicity.

\section{Treatment-experienced patients}

Lopinavir/ritonavir has been extensively evaluated versus other protease inhibitors in patients who are antiretroviral therapy experienced after virological failure on previous regimens. An open-label, randomized, multicenter trial of lopinavir/ritonavir with nevirapine and nucleoside reverse transcriptase inhibitors versus investigator-selected protease inhibitor(s) plus nevirapine and nucleoside reverse transcriptase inhibitors in 288 single protease inhibitorexperienced, non-nucleoside reverse transcriptase inhibitor (NNRTI)-naïve patients showed a greater proportion of patients with virologic suppression among those treated with lopinavir/ritonavir based regimens (57\% vs 33\%) (Pollard et al 2004).

A salvage trial of lopinavir/ritonavir combined with efavirenz in 57 patients who had failed at least two PI based regimens showed that virological response was correlated with the number of lopinavir mutations. Virologic suppression (HIV-RNA $<400$ copies/mL) was achieved in $91 \%$ of patients with $0-5$ mutations, $71 \%$ of the patients with 6-7 mutations and only $33 \%$ of patients with $8-10$ mutations (Kempf et al 2002). In PI and NNRTI-experienced patients lopinavir/ritonavir has been shown to produce a better virologic response than unboosted atazanavir (Cohen et al 2005). Lopinavir/ritonavir was shown to be superior to unboosted atazanavir in a study comparing the two agents in 300 patients who had failed a previous PI-based regimen. Virologic suppression (HIV-RNA $<50$ copies/mL) was seen in $54 \%$ of those taking lopinavir/ritonavir versus $38 \%$ of those taking unboosted atazanavir by intent to treat analysis $(p=0.008)$ (Cohen et al 2005). A randomized, non-blinded study (A5116) comparing combination therapy with lopinavir/ritonavir $533 \mathrm{mg} / 133 \mathrm{mg}$ twice daily and efavirenz $600 \mathrm{mg}$ once daily (in an NRTI-sparing regimen) with efavirenz and two NRTIs in patients $(n=236)$ who switched from PI or NNRTI-based regimens, showed that the combination of two NRTIs with efavirenz was more 
effective in achieving virologic suppression. The lopinavir/ ritonavir-based regimen in this study had significantly more toxicity-related discontinuations and shorter time to virologic failure ( $p=0.001)$ (Fischl et al 2007). Lopinavir/ritonavir was one of the four PIs administered to patients in the RESIST (Randomized Evaluation Strategic Intervention in Multi-Drug Resistant Patients with Tipranavir) studies. An analysis of the patients treated with tipranavir/ritonavir vs lopinavir/ritonavir showed a better virologic response (HIV-1 VL reduction of $\geq 1 \log 10$ from baseline) at 24 weeks in patients receiving tipranavir/ritonavir (40\%) versus lopinavir/ritonavir (21\%) (Cahn et al 2006; Gathe et al 2006) At 48 weeks, tipranavir/ritonavir therapy showed an improved virologic and immunological response rate compared with lopinavir/ritonavir in patients with virus exhibiting reduced susceptibility to lopinavir/ritonavir (Walmsley et al 2007).

In a randomized controlled trial (TITAN) comparing darunavir/ritonavir with lopinavir/ritonavir in treatment experienced, lopinavir naïve patients $(n=595)$, at week 48 a significantly greater number of patients treated with darunavir/ritonavir had HIV RNA $<400$ copies/mL (77\% vs $68 \%$ ). Fewer patients who were treated with darunavir/ ritonavir developed primary protease mutations or nucleoside analogue mutations as compared to lopinavir/ritonavir (Bongiovanni et al 2003).

The POWER 1 and 2 studies compared the safety and efficacy of boosted darunavir with that of the available protease inhibitors including lopinavir/ritonavir in a group of 255 highly antiretroviral experienced subjects. A pooled analysis of the two studies showed that at 48 weeks, patients in POWER 1 and 2 on darunavir/ritonavir achieved superior virologic suppression and immunologic response when compared with other currently available PIs including lopinavir/ ritonavir (Clotet et al 2007).

The MOTIVATE 1 and 2 studies showed that maraviroc, in combination with optimized background therapy (OBT), demonstrated superior virologic and immunologic treatment outcomes compared to OBT alone in treatment-experienced patients infected with CCR5-tropic HIV-1. A subanalysis of the MOTIVATE 1 and 2 studies showed that patients receiving maraviroc who had no lopinavir/ritonavir resistance, had an increased likelihood of achieving an undetectable viral load when lopinavir/ritonavir was included in the OBT (van der Ryst et al 2007). Lopinavir/ritonavir was also a part of the OBT in the BENCHMRK-1 and BENCHMRK-2 trials which were randomized double blind placebo controlled trials demonstrating the efficacy of the integrase inhibitor raltegravir when used in treatment experienced HIV-infected subjects who had failed antiretroviral therapies, and who had HIV virus resistant to at least one drug in each of the three available classes of oral antiretroviral therapies (Grinsztejn et al 2007).

\section{Monotherapy with lopinavir/ritonavir}

It has been hypothesized that monotherapy with a ritonavir-boosted HIV protease inhibitor may offer protection from the long-term toxicities of NRTIs while effectively maintaining long-term virological suppression (Sax 2005). A retrospective study $(\mathrm{n}=51)$ of virologically suppressed patients (HIV-RNA $<50$ copies/mL), who were switched to lopinavir/ritonavir monotherapy in an attempt to simplify treatment, found monotherapy with lopinavir/ritonavir to be a safe and effective treatment simplification approach especially in patients who were virologically suppressed on combination regimens containing lopinavir/ritonavir (Molto et al 2007).

MONARCH was a prospective, randomized, open label trial comparing lopinavir/ritonavir monotherapy $(n=83)$ with a standard regimen of lopinavir/ritonavir along with zidovudine and lamivudine $(\mathrm{n}=53)$ as an initial treatment in treatment-naive patients with HIV-RNA $<100,000$ copies/ml. Lopinavir/ritonavir monotherapy demonstrated lower rates of virological suppression compared with lopinavir/ritonavirNRTI therapy. At 48 weeks, in an intent-to-treat analysis, 53 of 83 patients $(64 \%)$ in the monotherapy group and 40 of 53 patients $(75 \%)$ in the triple-drug group achieved the primary virologic endpoint $(p=0.19)$. The on-treatment analysis indicated that 80 and $95 \%$ of patients reached the primary endpoint in the monotherapy and NRTI-lopinavir/ ritonavir, respectively ( $p<0.02$ ) (Delfraissy et al 2008).

A randomized, open-label, non-inferiority clinical trial in 205 virologically suppressed patients compared the continuation of triple therapy with lopinavir/ritonavir and two NRTIs versus lopinavir/ritonavir monotherapy with reintroduction of two nucleosides if virologic failure occurred with lopinavir/ ritonavir monotherapy (Pulido et al 2008). At 48 weeks, $94 \%$ of lopinavir/ritonavir monotherapy recipients survived without virologic failure compared with $90 \%$ of subjects receiving NRTI-lopinavir/ritonavir therapy. Episodes of low-level viremia were more common in patients receiving lopinavir/ritonavir monotherapy. In this study, 48 weeks of lopinavir/ritonavir monotherapy with reintroduction of nucleosides as needed was found to be non-inferior to continuation of two nucleosides and lopinavir/ritonavir in patients with prior stable suppression. 


\section{Pediatric studies}

Lopinavir/ritonavir has been evaluated in HIV-infected children $(n=100)$ between 3 months and 12 years of age in an open-label multicenter trial. Patients were randomized to either $230 \mathrm{mg}$ lopinavir $/ 57.5 \mathrm{mg}$ ritonavir $/ \mathrm{m}^{2}$ or $300 \mathrm{mg}$ lopinavir $/ 75 \mathrm{mg}$ ritonavir $/ \mathrm{m}^{2}$. Through 48 weeks of therapy, the proportion of patients who achieved and sustained an HIV-1 RNA $<400$ copies $/ \mathrm{mL}$ was $80 \%$ for antiretroviral naïve patients and $71 \%$ for antiretroviral experienced patients. The mean CD4+ lymphocyte count increase from baseline was 404 cells $/ \mathrm{mm}^{3}$ for antiretroviral naïve and 284 cells $/ \mathrm{mm}^{3}$ for antiretroviral experienced patients treated through 48 weeks. The drug was well tolerated overall, and there were very few adverse events with possible or probable relationship to the drug. A rash was noticed in 2 children and 2 others interrupted therapy intermittently due to elevations of amylase or hepatic enzymes. There were 2 premature discontinuations; 1 because of pancreatitis possibly related to lopinavir/ritonavir and the other due to an AIDS-associated malignancy (Saez-Llorens et al 2003).

\section{Resistance}

The pattern of resistance to lopinavir/ritonavir in antiretroviral therapy naïve patients has not been characterized (Abbott Laboratories). No clinical resistance was described in a clinical trial of antiretroviral therapy naïve adult patients with HIV infection (King et al 2004). In another trial, sustained clinical efficacy with no clinical resistance to lopinavir/ritonavir was observed in ART-naïve patients treated with lopinavir/ritonavir based regimens for up to 7 years (Murphy et al 2008). Similar results were seen in antiretroviral therapy naïve children who received lopinavir/ritonavir for 48 weeks (Saez-Llorens et al 2003). In a randomized double-blind trial of 653 antiretroviral therapy naïve patients who received lopinavir/ritonavir or nelfinavir plus lamivudine and stavudine for up to 96 weeks, no primary resistance mutations were seen in patients treated with lopinavir/ritonavir. In comparison, $45 \%$ of patients receiving nelfinavir showed evidence of resistance ( $p<0.001$ ) (King et al 2004). Resistance to lopinavir/ ritonavir has been shown to emerge in patients who have been exposed to other protease inhibitors. The mutations associated with decreased activity of lopinavir/ritonavir are 10/I/R/V, $20 \mathrm{M} / \mathrm{R}, 24 \mathrm{U}, 32 \mathrm{I}, 33 \mathrm{~F}, 46 \mathrm{I} / \mathrm{L}, 47 \mathrm{~V} / \mathrm{A}, 50 \mathrm{~V}$, $53 \mathrm{~L}, 54 \mathrm{~V} / \mathrm{L} / \mathrm{A} / \mathrm{M} / \mathrm{T} / \mathrm{S}, 63 \mathrm{P}, 71 \mathrm{~V} / \mathrm{Y}, 73 \mathrm{~S}, 82 \mathrm{~A} / \mathrm{FT} / \mathrm{S}, 84 \mathrm{~V}$, and 90M ( Kempf et al 2001). Lopinavir/ritonavir retains excellent antiviral efficacy despite the presence of 5 or fewer primary PI resistance mutations, but decreased efficacy is observed in the presence of higher numbers of mutations (Kempf et al 2002). The most common mutations associated with lopinavir resistance in antiretroviral therapy experienced adults are 46I/L. Mutations 32I and 47A are associated with high level resistance (Johnson et al 2006). The incidence of cross-resistance between lopinavir/ritonavir and other protease inhibitors is low. However, cross resistance between lopinavir/ritonavir and amprenavir may occur, and amprenavir resistance mutations may lead to decreased lopinavir/ritonavir effect. Mutations associated with lopinavir/ritonavir resistance are also associated with atazanavir resistance. In a study of dual PI therapy in a cohort of patients with an extensive pre-treatment history and highly variable pharmacokinetics, the absence of viral resistance mutations at $\mathrm{V} 82 \mathrm{~T} / \mathrm{A} / \mathrm{F} / \mathrm{I} / \mathrm{S}$ and $154 \mathrm{M} / \mathrm{V} / \mathrm{L}$ were highly predictive of therapeutic success with a regimen of saquinavir and lopinavir/ritonavir without reverse transcriptase inhibitors (von Hentig et al 2007). Phenotypic results can be used to guide interpretation of resistance and cross-resistance to lopinavir/ritonavir (Paulsen et al 2002). Lopinavir/ritonavir's high genetic barrier to genotypic resistance is one factor contributing to the enhanced forgiveness of non-adherence that this agent exhibits in comparison to other PIs (Shuter et al 2007).

\section{Drug interactions}

Protease inhibitors including lopinavir/ritonavir are substrates for CYP3A4 and CYP3A5 isoenzymes and may also inhibit or induce the activity of CYP3A4. Lopinavir/ritonavir may have significant interactions with drugs that are inducers or inhibitors of these enzymes and also with drugs that are substrates for the CYP3A4 and CYP3A5. Drugs that are contraindicated or require caution for coadministration with lopinavir/ritonavir are listed in Table 1. Interaction of lopinavir/ritonavir with other antiretroviral medications may require dosage adjustments. Table 2 summarizes the major interactions of lopinavir/ritonavir with other antiretroviral agents

\section{Adverse reactions}

Lopinavir/ritonavir-containing antiretroviral regimens are generally well tolerated. The most frequent side effects reported are diarrhea, nausea, and vomiting. These gastrointestinal adverse effects are generally mild to moderate (Abbott Laboratories). Diarrhea related to lopinavir/ritonavir therapy is reported by $15 \%-25 \%$ of patients. The incidence of diarrhea is dose-related and, with 
Table I Drug interactions with co-administration with lopinavir/ritonavir

\begin{tabular}{|c|c|}
\hline Rifampin & $\begin{array}{l}\text { Contraindicated - may lead to loss of virologic response and possible resistance to Pls } \\
\text { and other antiretroviral agents }\end{array}$ \\
\hline Rifabutin & Dose of rifabutin should be reduced $75 \%$ of the maximal dose \\
\hline Clarithromycin & Dose of clarithromycin should be reduced in patients with renal insufficiency \\
\hline Metronidazole & Disulfiram like reaction with lopinavir/ritonavir oral solution (contains $40 \%$ alcohol) \\
\hline Ergot derivatives & $\begin{array}{l}\text { Contraindicated - acute ergot toxicity with vasospasm and ischemia of extremities } \\
\text { and other tissues }\end{array}$ \\
\hline Cisapride & Contraindicated - cardiac arrhythmias \\
\hline St. John's Wort & $\begin{array}{l}\text { Contraindicated - may lead to loss of virologic response and possible resistance to Pls } \\
\text { and other antiretroviral agents }\end{array}$ \\
\hline Trazodone & Increased levels of trazodone \\
\hline Lovastatin, simvastatin & Potential for myopathy including rhabdomyolysis \\
\hline Pimozide & Contraindicated - cardiac arrhythmias \\
\hline Benzodiazepines & Contraindicated - prolonged sedation and respiratory depression \\
\hline Phenytoin & Decreased $A \cup C$ and $C_{\text {trough }}$ of both drugs \\
\hline Methadone & Dosage of methadone may need to be increased \\
\hline Voriconazole & Lack of data on co-administration \\
\hline Fluconazole, ketoconazole & Avoid high doses of these drugs with lopinavir/ritonavir \\
\hline Fluticasone & Cushing's syndrome, adrenal suppression, osteoporosis \\
\hline Amiodarone, lidocaine, quinidine & Increased levels of these agents \\
\hline Propafenone & Contraindicated - cardiac arrhythmias \\
\hline Flecainide & Contraindicated - cardiac arrhythmias \\
\hline PDE5 inhibitors (sidenafil, tadafil, vardenafil) & hypotension, syncope, priapism and visual changes \\
\hline Warfarin & May require more frequent monitoring of INR and dosage adjustments of warfarin ritonavir \\
\hline
\end{tabular}

Abbreviations: INR, international normalized ratio; $\mathrm{PI}, \mathrm{HIV}-\mathrm{I}$ protease inhibitor.

the old capsule formulation, occurred more frequently with the lopinavir/ritonavir $800 / 200 \mathrm{mg}$ once-daily dose than lopinavir/ritonavir 400/100 mg twice-daily dose (Murphy et al 2001). The newer tablet formulation is better tolerated than the capsule formulation. The incidence of diarrhea due to lopinavir/ritonavir is higher than that due to atazanavir (Johnson et al 2005). However, patients who switched therapy from another PI-based regimen to lopinavir/ ritonavir based regimen showed an overall improvement in side effects and health related quality of life (Luo et al 2004). Immune reconstitution syndrome has been reported in patients treated with antiretroviral regimens containing lopinavir/ritonavir, manifested as an inflammatory response to indolent opportunistic infections. Serious side effects of lopinavir/ritonavir therapy are unusual. Pancreatitis has been seen rarely in patients on lopinavir/ritonavir therapy, and may have been related to marked elevations in triglyceride levels. Patients with a known history of pancreatitis are more likely to develop pancreatitis on lopinavir/ritonavir therapy (Oldfield and Plosker 2006). Lopinavir/ritonavir can cause transient elevations in transaminase levels, but these are usually not clinically significant. The incidence of severe hepatic events in patients receiving lopinavir/ritonavir is very low. Hepatitis $\mathrm{C}$ coinfection and baseline elevations in transaminases may be associated with severe liver events in lopinavir/ritonavir recipients (Palacios et al 2006). Patients receiving lopinavir/ritonavir therapy with underlying liver disease should be monitored closely with frequent measures of hepatic function (Abbott Laboratories). Like other members of the protease inhibitor class, lopinavir/ritonavir may cause significant lipid elevations and fat redistribution. Hypertriglyceridemia (triglycerides $>750 \mathrm{mg} / \mathrm{dL}$ ) and hypercholesterolemia (total cholesterol $>300 \mathrm{mg} / \mathrm{dL}$ ) were the most frequently observed laboratory abnormalities in lopinavir/ritonavir recipients in clinical trials and may be the reason for discontinuation of therapy in some patients (Bongiovanni et al 2005). Increases in total cholesterol and triglycerides are seen within the first month of starting therapy and are relatively stable after this time (Lafeuillade et al 2004). The lipid elevations due to lopinavir/ritonavir generally respond to lipid lowering therapy. Some trials have found that the lipid derangements 
Table 2 Drug interactions of lopinavir/ritonavir with other antiretroviral agents

\begin{tabular}{|c|c|c|}
\hline Drug class, agents & Drug interaction & Clinical significance \\
\hline \multicolumn{3}{|l|}{ NNRTIs } \\
\hline Efavirenz & $\downarrow$ lopinavir concentration & $\begin{array}{l}\text { Increased dose of lopinavir/ritonavir may be needed } \\
\text { Lopinavir/ritonavir should not be used once daily with efavirenz }\end{array}$ \\
\hline Nevirapine & $\downarrow$ lopinavir concentration & $\begin{array}{l}\text { Increased dose of lopinavir/ritonavir may be needed } \\
\text { Lopinavir/ritonavir should not be used once daily with nevirapine }\end{array}$ \\
\hline Delavirdine & $\uparrow$ lopinavir concentration & Dose not established for use in combination \\
\hline \multicolumn{3}{|l|}{ NRTIs } \\
\hline Abacavir & $\downarrow$ abacavir concentration & Unknown \\
\hline Tenofovir & $\uparrow$ tenofovir concentration & Monitor for adverse reactions of tenofovir \\
\hline Zidovudine & $\downarrow$ zidovudine concentration & Unknown \\
\hline \multicolumn{3}{|l|}{ Pls } \\
\hline Amprenavir & $\begin{array}{l}\downarrow \text { lopinavir concentration } \\
\uparrow \text { amprenavir concentration }\end{array}$ & $\begin{array}{l}\text { Lopinavir/ritonavir should not be administered in once-daily } \\
\text { combination with amprenavir }\end{array}$ \\
\hline Fosamprenavir/ritonavir & $\begin{array}{l}\downarrow \text { lopinavir concentration } \\
\downarrow \text { amprenavir concentration }\end{array}$ & $\begin{array}{l}\text { Increased rate of adverse effects observed } \\
\text { Appropriate dose in combination not established }\end{array}$ \\
\hline Indinavir & $\uparrow$ indinavir concentration & $\begin{array}{l}\text { Decrease indinavir dose to } 600 \text { bid when used with } \\
\text { lopinavir/ritonavir } 400 / 100 \mathrm{mg} \text { bid } \\
\text { Once-daily lopinavir/ritonavir has not been studied with indinavir }\end{array}$ \\
\hline Nelfinavir & $\begin{array}{l}\downarrow \text { lopinavir concentration } \\
\uparrow \text { nelfinavir concentration } \\
\uparrow \text { M8 metabolite of nelfinavir }\end{array}$ & $\begin{array}{l}\text { Lopinavir/ritonavir should not be used once daily in combination } \\
\text { with nelfinavir }\end{array}$ \\
\hline Ritonavir & $\uparrow$ lopinavir concentration & Appropriate doses of additional ritonavir not established \\
\hline Saquinavir & $\uparrow$ saquinavir concentration & $\begin{array}{l}\text { Saquinavir dose should be } 1000 \mathrm{mg} \text { bid with lopinavir/ritonavir } \\
400 / 100 \mathrm{mg} \text { bid } \\
\text { Lopinavir/ritonavir once daily has not been studied } \\
\text { with saquinavir }\end{array}$ \\
\hline Tipranavir & $\downarrow$ lopinavir $\mathrm{AUC}$ and $\mathrm{C}_{\min }$ & $\begin{array}{l}\text { Lopinavir/ritonavir should not be used with tipranavir } 500 \mathrm{mg} \\
\text { bid/ritonavir } 200 \mathrm{mg} \text { bid }\end{array}$ \\
\hline
\end{tabular}

Abbreviations: NRTI, nucleoside reverse transcriptase inhibitors; NNRTI, non-nucleoside reverse transcriptase inhibitor; PI, HIV-I protease inhibitor.

caused by lopinavir/ritonavir were more severe than those caused by other PIs such as atazanavir, atazanavir/ritonavir, or nelfinavir (Walmsley et al 2002; Cohen et al 2005), but other trials reported similar elevations in triglycerides and cholesterol in lopinavir/ritonavir recipients as compared to subjects receiving other PIs (Dragsted et al 2005). PIs including lopinavir/ritonavir have been associated with insulin resistance, new onset diabetes, and worsening of pre-existing diabetes requiring hypoglycemic agents in some patients. Hyperglycemia may persist in patients even after discontinuation of lopinavir/ritonavir. Fat redistribution including central obesity, dorsocervical fat enlargement (buffalo hump), peripheral wasting, facial wasting, breast enlargement, and cushingoid appearance have been observed in patients receiving antiretroviral therapy including lopinavir/ritonavir. However, a causal relationship has not been established (Abbott Laboratories). There is evidence to suggest that fat redistribution in less likely to occur in individuals receiving unboosted atazanavir (Haerter et al 2004). Less common adverse effects of lopinavir/ritonavir therapy include allergic reaction, asthenia, malaise, headache, myalgias, arthralgias, myocardial infarction, seizures, and lactic acidosis.

\section{Conclusion}

Lopinavir/ritonavir played a major role in ushering in the era of boosted PI therapy, and in offering the first good option to patients who had failed prior therapy. It has proven efficacious in the treatment of HIV infection in both ART naive as well as experienced patients. Despite the availability of newer agents, the wealth of clinical experience with this important medication ensures it a prominent place in the antiretroviral treatment armamentarium for years to come. 


\section{Acknowledgments}

This work was supported, in part, by the Clinical Core of the Center for AIDS Research at the Albert Einstein College of Medicine and Montefiore Medical Center funded by the National Institutes of Health (NIH AI-51519).

\section{Disclosures}

Dr Shuter has received research grant support from Abbott Laboratories through their investigator-initiated research program.

\section{References}

Abbott Laboratories. Kaletra ${ }^{\circledR}$ (lopinavir/ritonavir) tablets and oral solution. Prescribing information [online]. Accessed 20 March 2008. URL: http://www.kaletra.com

Bongiovanni M, Bini T, Adorni F, et al. 2003. Virological success of lopinavir/ritonavir salvage regimen is affected by an increasing number of lopinavir/ritonavir-related mutations. Antivir Ther, 8:209-14.

Bongiovanni M, Cicconi P, Landonio S, et al. 2005. Predictive factors of lopinavir/ritonavir discontinuation for drug-related toxicity: results from a cohort of 416 multi-experienced HIV-infected individuals. Int $J$ Antimicrob Agents, 26:88-91.

Cahn P, Villacian J, Lazzarin A, et al. 2006. Ritonavir-boosted tipranavir demonstrates superior efficacy to ritonavir-boosted protease inhibitors in treatment-experienced HIV infected patients: 24 weeks results of RESIST 2 trial. Clin Infect Dis, 43:1347-56.

Clotet B, Bellos N, Molina JM, et al. 2007. Efficacy and safety of darunavirritonavir at week 48 in treatment-experienced patients with HIV-1 infection in POWER 1 and 2: a pooled subgroup analysis of data from two randomized trials. Lancet, 369:1169-78.

Cohen C, Nieto-Cisneros L, Zala C, et al. 2005. Comparison of atazanavir with lopinavir/ritonavir in patients with prior protease inhibitor failure: a randomized multinational trial. Curr Med Res Opin, 21:1683-92.

Crommentuyn KML, Mulder JW, Mairuhu ATA. 2004. The plasma and intracellular steady-state pharmacokinetics of lopinavir/ritonavir in HIV-1 infected patients. Antivir Ther, 9:779-85.

Cunha AM, Hagemann CC, Simones RS, et al. 2007. Effects of lopinavir-ritonavir combined therapy during the rat pregnancy. Morphological and biochemical aspects. Eur J Obstet, Gynecol Reprod Biol, 133:60-3.

Deeks SG, Smith M, Holodniy M, et al. 1997. HIV-1 protease inhibitors. A review for clinicians. JAMA, 277:145-53.

Delfraissy JF, Flandre P, Delaugerre C, et al. 2008. Lopinavir/ritonavir monotherapy or plus zidovudine and lamivudine in antiretroviral-naïve HIV-infected patients. AIDS, 22:385-93.

Dragsted UB, Gerstoft J, Youle M, et al. 2005. A randomized trial to evaluate lopinavir/ritonavir versus saquinavir/ritonavir in HIV-1-infected patients: the MaxCmin2 trial. Antivir Ther, 10:735-43.

Eron J Jr, Yeni P, Gathe J Jr, et al. 2006. The KLEAN study of fosamprenavirritonavir versus lopinavir-ritonavir, each in combination with abacavirlamivudine, for initial treatment of HIV infection over 48 weeks: a randomised non-inferiority trial. Lancet, 367:476-82.

FDA/Center for Drug Evaluation and Research. Drugs@FDA [online]. Accessed 25 March 2008. URL: http://www.accessdata.fda.gov/scripts/ cder/drugsatfda.

Fischl MA, Collier AC, Mukherjee AL, et al. 2007. Randomized open-label trial of two simplified, class-sparing regimens following a first suppressive three or four-drug regimen. AIDS, 21:325-33.

Flexner C. 1998. HIV-Protease inhibitors. N Engl J Med, 338:1281-93.

Gathe J Jr, Cooper DA, Farthing C, et al. 2006. Efficacy of protease inhibitors tipranavir plus ritonavir in treatment-experienced patients: 24 week analysis from RESIST-1 trial. Clin Infect Dis, 43:1337-46.
Gazzard B on behalf of BHIVA Writing Committee. British HIV Association Guidelines for treatment of HIV infected adults with antiretroviral therapy (2006). [Online] [Accessed 20March 2008] URL: http://www. bhiva.org.

Gonzalez-Requena D, Nunez M, Jimenez-Nacher I, et al. 2004. Short communication: liver toxicity of lopinavir-containing regimens in HIV-infected patients with or without hepatitis C coinfection. AIDS Res Hum Retroviruses, 20:698-700.

Grinsztejn B, Nguyen BY, Katlama C, et al. 2007. Safety and efficacy of the HIV-1 integrase inhibitor raltegravir (MK-0518) in treatmentexperienced patients with multidrug-resistant virus: a phase II randomised controlled trial. Lancet, 369:1261-9.

Gulick RM, Hu XJ, Fiscus SA, et al. 2000. Randomized study of saquinavir with ritonavir or nelfinavir together with delavirdine, adefovir, or both in human immunodeficiency virus-infected adults with virologic failure on indinavir: AIDS Clinical Trials Group Study 359. J Infect Dis, 182:1375-84.

Haerter G, Manfras BJ, Mueller M, et al. 2004. Regression of lipodystrophy in HIV-infected patients under therapy with the new protease inhibitor atazanavir. AIDS, 18:952-5.

Hammer SM, Bassett R, Squires KE, et al. 2003. A randomized trial of nelfinavir and abacavir in combination with efavirenz and adefovir dipivoxil in HIV-1-infected persons with virological failure receiving indinavir. Antivir Ther, 8:507-18.

Hammer SM, Saag MS, Schechter M, et al. 2006. Treatment for Adult HIV infection. 2006 recommendations of the International AIDS SocietyUSA Panel. JAMA, 296: 827-43.

Johnson MA, Gathe JC Jr, Podzamczer D, et al. 2006. A once-daily lopinavir/ritonavir-based regimen provides noninferior antiviral activity compared with a twice-daily regimen. J Acquir Immune Defic Syndr, 43:153-60.

Johnson M, Grinsztejn B, Rodriguez C, et al. 2005. Atazanavir plus ritonavir or saquinavir, and lopinavir/ritonavir in patients experiencing multiple virological failures. AIDS, 19:685-94.

Johnson VA, Brun-Vezinet F, Clotet B, et al. 2006. Update of the Drug Resistance Mutations in HIV-1: Fall 2006. Top HIV Med, 14:125-30.

Kempf DJ, Isaacson JD, King MS, et al. 2001. Identification of genotypic changes in human immunodeficiency virus protease that correlate with reduced susceptibility to the protease inhibitor lopinavir among viral isolates from protease inhibitor-experienced patients. $J$ Virol, 75:7462-9.

Kempf DJ, Isaacson JD, King MS, et al. 2002. Analysis of virologic response with respect to baseline viral phenotype and genotype in protease inhibitor-experienced HIV-infected patients receiving lopinavir/ ritonavir. Antivir Ther, 7:165-74.

King MS, Bernstein BM, Walmsley SL, et al. 2004. Baseline HIV-1 RNA level and CD4 cell count predict time to loss of virologic response to nelfinavir, but not lopinavir/ritonavir, in antiretroviral therapy - naive patients. J Infect Dis, 190:280-4.

Klein CE, Chiu YL, Awni W, et al. 2007. The tablet formulation of lopinavir/ ritonavir provides similar bioavailability to the soft-gelatin capsule formulation with less pharmacokinetic variability and diminished food effect. J Acquir Immune Defic Syndr, 44:401-10.

Kumar GN, Dykstra J, Roberts EM, et al. 1999. Potent inhibition of the cytochrome P450 3A-mediated human liver microsomal metabolism of a novel HIV protease inhibitor by ritonavir: A prospective drug-drug interaction. Drug Metab Dispos, 27:902-8.

Kumar, GN, Jayanti VK, Johnson MK, et al. 2004. Metabolism and disposition of the HIV-1 protease inhibitor lopinavir (ABT-378) given in combination with ritonavir in rats, dogs and humans. Pharm Res, 21:1622-30

Lafeuillade A, Hittinger G, Philip G, et al. 2004. Metabolic evaluation of HIV-infected patients receiving a regimen containing lopinavir/ritonavir (Kaletra). HIV Clin Trials, 5:392-8.

Letendre SL, van der Brande G, Hermes A, et al. 2007. Lopinavir and ritonavir reduces the HIV RNA level in cerebrospinal fluid. Clin Infect Dis, 45:1511-7. 
Lim ML, Min SS, Eron JJ, et al. 2004. Coadministration of lopinavir/ritonavir and phenytoin results in two-way drug interaction through cytochrome P-450 induction. J Acquir Immune Defic Syndr, 36:1034-40.

Luo MP, Shen Y, Rode R, McMillan F, et al. 2004. Substitution to lopinavir/ ritonavir is associated with improved patient-reported fatigue in HIV positive patients experiencing side effects related to their protease inhibitor/ non-nucleoside reverse transcriptase inhibitor. Value Health, 7:360.

Madruga JV, Berger D, McMurchie M, et al. 2007. Efficacy and safety of darunavir-ritonavir compared with that of lopinavir-ritonavir at 48 weeks in treatment-experienced, HIV-infected patients in TITAN: a randomised controlled phase III trial. Lancet, 370:49-58.

McCance-Katz EF, Rainey PM, Friedland G, et al. 2003. The protease inhibitor lopinavir-ritonavir may produce opiate withdrawal in methadone-maintained patients. Clin Infect Dis, 37:476-82.

Molina JM, Andrade-Villanueva J, Echevarria J, et al. 2008. Efficacy and safety of once-daily atazanavir/ritonavir compared to twice-daily lopinavir/ritonavir, each in combination with tenofovir and emtricitabinein ARV-naive HIV-1-infected subjects: The CASTLE Study, 48-week results [abstract]. 15th Conference on Retroviruses and Opportunistic Infections, Boston, Feb 3-6 2008, Abstract 37.

Molto J, Santos JR, Negredo E, et al. 2007. Lopinavir/ritonavir monotherapy as a simplification strategy in routine clinical practice. J Antimicrob Chemother, 60:436-9.

Murphy RL, Brun S, Hicks CB, et al. 2001. ABT-378/ritonavir puls stavudine and lamivudine for the treatment of antiretroviral naïve adults with HIV infection: 48 week results. AIDS, 15:F1-9.

Murphy RL, da Silva BA, Hicks CB, et al. 2008. Seven-year efficacy of a lopinavir/ritonavir-based regimen in antiretroviral-naïve HIV-1-infected patients. HIV Clin Trials, 9:1-10.

Oldfield V, Plosker GL. 2006. Lopinavir/ritonavir. A review of its use in the management of HIV infection. Drugs, 66:1275-99.

Ortiz R, Dejesus E, Khanlou H, et al. 2008. Efficacy and safety of oncedaily darunavir/ritonavir versus lopinavir/ritonavir in treatment-naive HIV-1-infected patients at week 48. AIDS, 22:1389-97.

Palacios R, Vergara S, Rivero A, et al. 2006. Lower incidence of severe liver events in HIV patients with and without hepatitis $\mathrm{C}$ or B coinfection receiving lopinavir/ritonavir. HIV Clin Trials, 7:319-23.

Panel on clinical practices for treatment of HIV infection. Guidelines for use of antiretroviral agents in HIV infected adults and adolescents [online]. Accessed 20 March 2008. URL: http://AIDSinfo.nih.gov.

Parkin NT, Chappey C, Petrpoulos CJ. 2003. Improving lopinavir genotype algorithm through phenotype correlations: novel mutation patterns and amprenavir cross-resistance. AIDS, 17:955-61.

Paterson DL, Swindells S, Mohr J, et al. 2000. Adherence to protease inhibitor therapy and outcomes in patients with HIV infection. Ann Intern Med, 133:21-30.

Paulsen D, Liao Q, Fusco G, et al. 2002. Genotypic and phenotypic cross-resistance patterns to lopinavir and amprenavir in protease inhibitor-experienced patients with HIV viremia. AIDS Res Hum Retroviruses, 18:1011-9.

Pollard RB, Thompson M, Hicks M, et al. 2004. Phase 3 comparison of lopinavir/ritonavir vs investigator-selected protease inhibitor in single PI experienced NNRTI-naïve patients: 48 week results of study M98-888 [poster]. 7th International Congress on Drug Therapy in HIV Infection, Glasgow, Nov 14-18 2004, Abstract No PI.3.2 plus poster.
Pulido F, Arribas JR, Delgado R, et al. 2008. Lopinavir-ritonavir monotherapy versus lopinavir-ritonavir and two nucleosides for maintenance therapy of HIV. AIDS, 22:F1-9.

Rana KZ, Dudley MN. 1999. Human immunodeficiency virus protease inhibitors. Pharmacotherapy, 19:35-59.

Riddler SA, Haubrich R, DiRenzo G, et al. 2008. Class-sparing regimens for initial treatment of HIV-1 infection. N Engl J Med, 358:2095-2106.

Saez-Llorens X, Violari A, Deetz Co, et al. 2003. Forty-eight-week evaluation of lopinavir/ritonavir, a new protease inhibitor, in human immunodeficiency virus-infected children. Pediatr Infect Dis $J$, 22:216-23.

Sax PE. 2005. Top stories of 2004. Back to the future: lopinavir/ritonavir as "monotherapy". AIDS Clin Care, 17:4.

Sham HL, Kempf DJ, Molla A, et al. 1998. ABT-378, a highly potent inhibitor of the human immunodeficiency virus protease. Antimicrob Agents Chemother, 42:3218-24.

Shuter JS, Sarlo J, Kanmaz TJ, et al. 2007. HIV-infected patients receiving lopinavir/ritonavir-based antiretroviral therapy achieve high rates of virologic suppression despite adherence rates less than $95 \%$. J Acquir Immune Defic Syndr, 45:4-8.

Taburet AM, Raguin G, Le Tiec C, et al. 2004. Interactions between amprenavir and the lopinavir-ritonavir combination in heavily pretreated patients infected with human immunodeficiency virus. Clin Pharmacol Ther, 75:310-23.

Third World Network. As AIDS drugs fail, few have any alternative [online]. August 23, 2006. URL: http://www.twnside.org.sg/title2/health.info/ twninfohealth032.htm.

van der Ryst E, Cooper D, Konourina I, et al. 2007. Efficacy of Maraviroc in combination with at least one other potent new antiretroviral drug: 24-week combined analysis of the MOTIVATE 1 and 2 studies [abstract]. 4th IAS Conference on HIV Pathogenesis, Treatment and Prevention. Abstract no. WEPEB115LB.

von Hentig N, Babacan E, Staszewski S, et al. 2007. Predictive factors for response to a boosted dual HIV-protease inhibitor therapy with saquinavir and lopinavir in extensively pre-treated patients. Antivir Ther, 12:1237-46.

Walmsley S, Bernstein B, King M, et al. 2002. Lopinavir-ritonavir versus nelfinavir for the initial treatment of HIV infection. $N$ Engl J Med, 346:2039-46.

Walmsley S, Ruxrungtham L, Slim J, et al. 2007. Saquinavir/r (SQV/r) bid versus lopinavir/r (LPV/r) bid, plus emtricitabine/tenofovir (FTC/ TDF) qd as initial therapy in HIV-1-infected patients: The Gemini Study. 11th European AIDS Conference. Madrid, Spain. October 24-27, 2007.

Walmsley SL, Cotte L, Rusconi S, et al. 2007. Treatment response to ritonavir-boosted tipranavir versus ritonavir-boosted lopinavir in HIV-1 patients with higher lopinavir mutation scores. AIDS, $21: 2245-8$

World Health Organization. 2006. Antiretroviral therapy for HIV infection in adults and adolescents: Recommendations for a public health approach. Geneva: WHO Press.

Yilmaz A, Stahle L, Hagberg L, et al. 2004.Cerebrospinal fluid and plasma HIV-1 RNA levels and lopinavir concentrations following lopinavir/ ritonavir regimen. Scand J Infect Dis, 36:823-8. 
